\title{
ESTIMATIVA DA RADIAÇÃO SOLAR GLOBAL PARA A REGIÃO DE LAVRAS, MG
}

\section{ANTONIO AUGUSTO AGUILAR DANTAS ${ }^{1}$ LUIZ GONSAGA DE CARVALHO ${ }^{2}$ ELIZABETH FERREIRA ${ }^{3}$}

\begin{abstract}
RESUMO - Realizou-se este trabalho com o objetivo de determinar as constantes "a" e "b" do modelo linear de Angstrom para estimativa da radiação solar global em Lavras, MG. O trabalho foi desenvolvido na Estação Climatológica Principal de Lavras (ECPINMET/UFLA), localizada no campus da Universidade Federal de Lavras, no período de dezembro de 2001 a novembro de 2002, a partir de dados diários de insolação e de dados de radiação solar global. Por análise de regressão, obtiveram-se as constantes a e b, parâmetros de regressão do modelo que caracterizam a transmitân-
\end{abstract}

cia atmosférica. A equação obtida com os valores médios analisados, $\mathrm{Qg} / \mathrm{Qt}=0,23+0,49 \mathrm{n} / \mathrm{N}$ apresentou coeficiente de determinação médio de 0,89 . Os resultados foram menores que os sugeridos pela literatura, obtidos em função da latitude do local onde não são disponíveis os coeficientes. De acordo com os resultados encontrados, pode-se indicar os valores de 0,23 e 0,49 , respectivamente, como " a" e "b", para serem utilizados na equação de Angstrom nas estimativas de radiação solar global para Lavras, MG.

TERMOS PARA INDEXAÇÃO: Radiação solar global, modelo linear de Angstrom, coeficientes.

\section{GLOBAL SOLAR RADIATION ESTIMATION IN LAVRAS REGION, MG}

\begin{abstract}
The objective of this work was the determination of the "a" and " $b$ " constants of the Angstrom linear model in order to estimate the global solar radiation in Lavras, MG. The work was carried out in the Climatological Station of Lavras (ECP/INMET/UFLA), at the Federal University of Lavras, from December 2001 to November 2002, through insolation daily data and global solar radiation daily records. The "a" and " b" constants, that express
\end{abstract}

the atmospheric transmitance, were obtained by regression analysis of those data. The obtained equation, $\mathrm{Qg} / \mathrm{Qt}=0,23+0,49$ presented a determination coefficient of 0,89 . The results are smaller than those suggested by the recomendations that uses the local latitude. According to the results, its possible to indicate the values of 0,23 and 0,49 to be used as the "a" and " $b$ " constants on the Angstrom equation to estimate the global solar radiation in Lavras, MG.

INDEX TERMS: Global solar radiation, Angstrom linear model, coefficients.

\section{INTRODUÇÃO}

Trabalhos de pesquisa por meio dos quais podese estimar o potencial de radiação solar disponível à superfície da Terra são plenamente justificados, em razão da sua real importância para a execução de projetos de irrigação, produção agrícola, aproveitamento de energia, conservação de alimentos, entre outros.
O levantamento da disponibilidade de energia solar à superfície deveria ser feito por meio de medições diretas, com uso de piranômetros. Entretanto, tais medições exigem o uso de registradores ou sistemas de aquisição de dados, e pessoal habilitado, o que dificulta e eleva o custo dessas informações. Uma alternativa para superar tal impasse é o uso de modelos teóricos, ajustados para o local de interesse.

1. Zootecnista, Dsc, Área de Agrometeorologia, Departamento de Engenharia, UNIVERSIDADE FEDERAL DE LAVRAS/UFLA, Caixa Postal 37 - 37200-000 - Lavras, MG.

2. Engenheiro Agrícola, Msc, Área de Agrometeorologia, Departamento de Engenharia, UFLA.

3. Engenheiro Agrícola, Dsc, Área de Fotointerpretação, Departamento de Engenharia, UFLA. 
Entre as expressões empíricas simples que permitem conhecer a distribuição espacial da radiação solar, destaca-se o modelo de Angstrom (TUBELIS e NASCIMENTO, 1980), qual seja:

$$
\frac{Q g}{Q t}=a+b \frac{n}{N}
$$

em que:

Qg = radiação solar global recebida na superfície terrestre (ly/dia).

$\mathrm{Qt}$ = radiação total recebida em uma superfície plana e horizontal, na ausência da atmosfera (ly/dia).

$\mathrm{n}$ = insolação diária, (horas)

$\mathrm{N}=$ comprimento astronômico do dia, (horas)

$\mathrm{a}$ e $\mathrm{b}=$ parâmetros de regressão do modelo que caracterizam a transmitância atmosférica.

Para os locais entre as latitudes $0^{\circ}$ e $60^{\circ}$, em que essas constantes não são conhecidas, pode-se utilizar os valores propostos por Glover e McCullosh, citados por Tubelis e Nascimento (1980):

$$
\mathrm{a}=0,29 \cos \phi \text {, sendo } \phi \text { a latitude local e } \mathrm{b}=0,52
$$

No Brasil, foram determinados os valores desses coeficientes para vários locais no Estado de São Paulo (CERVELLINI et al., 1966; OMETTO, 1968; FOSTER e SANTOS, 1985; FOSTER et al., 1985; LAPERUTA FILHO et al., 1997), no Estado de Minas Gerais (ALVES, 1981), Rio Grande do Norte (ESPÍNOLA SOBRINHO e IZÍDIO, 1993), Santa Catarina (BRAGA et al., 1987), Pará (CARDON e MALTEZ, 1987), além de outros Estados (TUBELIS e NASCIMENTO, 1980).

Neste trabalho objetivou-se a determinação dos parâmetros "a" e "b" do modelo linear de Angstrom para Lavras, Estado de Minas Gerais.

\section{MATERIAL E MÉTODOS}

O trabalho foi desenvolvido a partir de dados coletados na Estação Climatológica Principal de Lavras (ECP-INMET/UFLA), localizada no campus da Universidade Federal de Lavras, em Lavras, MG, no período de dezembro de 2001 a novembro de 2002. O local apresenta as seguintes coordenadas geográficas: latitude $21^{\circ} 13^{\prime} 32^{\prime \prime} \mathrm{S}$; longitude $45^{\circ} 58^{\prime} 45^{\prime \prime} \mathrm{W}$ Gr., datum SAD 69. A altitude é de $918,8 \mathrm{~m}$ e o clima é Cwa, conforme a classificação climática de Köppen.
A radiação total foi obtida, segundo Sellers (1974), por:

$$
Q t=\frac{1440 \cdot S}{\pi} \cdot\left(\frac{\bar{D}}{D}\right)^{2} \cdot[H \cdot \operatorname{sen} \phi \cdot \operatorname{sen} \delta+\cos \phi \cdot \cos \delta \cdot \operatorname{sen} H]
$$

em que:

$S=$ constante solar

$(\overline{\mathrm{D}} / \mathrm{D})^{2}=$ correção devida à variação da distância Terra-Sol

$\mathrm{H}=$ comprimento do meio dia solar, estimado por:

$H=\operatorname{arc} \cos (-\tan \phi . \tan \delta)$

$\phi$ é a latitude do local.

$\delta=$ declinação solar (graus)

A estimativa do comprimento astronômico do dia (N) foi obtida por:

$$
\mathrm{N}=2 \mathrm{H} / 15
$$

Os dados foram submetidos à análise de regressão, considerando-se $Q g / Q t$ como variáveis dependentes e $n / N$ como variáveis independentes.

\section{RESULTADOS E DISCUSSÃO}

A equação obtida com os valores médios analisados apresentou coeficiente de determinação de 0,89 :

$$
\mathrm{Qg} / \mathrm{Qt}=0,23+0,49 \mathrm{n} / \mathrm{N}
$$

Para aumentar a precisão da estimativa, podemse utilizar os valores mensais dos coeficientes apresentados no Quadro 1.

\section{CONCLUSÕES}

De acordo com os resultados encontrados, podem-se indicar os valores de 0,23 e 0,49 , respectivamente como "a" e "b", a serem utilizados na equação de Angstrom para estimar a radiação solar global em Lavras, Estado de Minas Gerais.

Esses resultados são menores do que aqueles propostos por Glover e McCullosh, citados por Tubelis e Nascimento (1980), que apresentam os valores dos coeficientes em função da latitude do local, para serem utilizados em localidades onde não são disponíveis os coeficientes. 
QUADRO 1 - Valores da constantes "a" e "b" da Equação de Angstrom e coeficiente de determinação.

\begin{tabular}{lccc}
\hline \multicolumn{1}{c}{ Meses } & Constante"a" & Constante"b" & Coeficiente de Determinação \\
\hline Dezembro 2001 & 0,22 & 0,50 & 0,96 \\
\hline Janeiro 2002 & 0,20 & 0,55 & 0,90 \\
\hline Fevereiro 2002 & 0,27 & 0,43 & 0,89 \\
\hline Março 2002 & 0,30 & 0,34 & 0,80 \\
\hline Abril 2002 & 0,22 & 0,50 & 0,84 \\
\hline Maio 2002 & 0,28 & 0,41 & 0,75 \\
\hline Junho 2002 & 0,18 & 0,52 & 0,85 \\
\hline Julho 2002 & 0,18 & 0,54 & 0,87 \\
\hline Agosto 2002 & 0,21 & 0,50 & 0,93 \\
\hline Setembro 2002 & 0,21 & 0,52 & 0,93 \\
\hline Outubro 2002 & 0,19 & 0,56 & 0,97 \\
\hline Novembro 2002 & 0,24 & 0,48 & 0,93 \\
\hline Período & 0,23 & 0,49 & 0,89 \\
\hline
\end{tabular}

\section{AGRADECIMENTOS}

À FAPEMIG, Processo CAG-2723/97 pelo financiamento do Projeto.

\section{REFERÊNCIAS BIBLIOGRÁFICAS}

ALVES, A. R. Irradiância solar global em superfícies de diferentes inclinações e azimutes, para Viçosa, MG. 1981. 92 f. Dissertação (Mestrado em Engenharia Agrícola) - Universidade Federal de Viçosa, Viçosa, 1981

BRAGA, H. J.; SILVA, L. M.; LEITE, G. B. Determinação preliminar dos parâmetros $\underline{\mathrm{a}} \mathrm{e} \underline{\mathrm{b}}$ da equação de Angstrom para seis localidades de Santa Catarina. In: CONGRESSO BRASILEIRO DE AGROMETEOROLOGIA, 5., 1987, Belém. Anais... Belém: [s.n.], 1987. p. 199-200.

CARDON, D. A.; MALTEZ, M. G. L. Influência da nebulosidade na estimativa da radiação global em Belém. In: CONGRESSO BRASILEIRO DE AGROMETEOROLOGIA, 5., 1987, Belém. Anais... Belém: [s.n.], 1987. p. 202-210.
CERVELLINI, A.; SALATI, E.; GODOY, H. Estimativa da distribuição da energia solar no Estado de São Paulo. Bragantia, Campinas, v. 23, p. 31-39, 1966.

ESPÍNOLA SOBRINHO, J.; IZÍDIO, L. R. Determinação dos parâmetros "a" e "b" da equação de Angstron para estimativa da irradiação solar global em Mossoró-RN. In: CONGRESSO BRASILEIRO DE AGROMETEOROLOGIA, 8., 1993, Porto Alegre. Resumos... Santa Maria: [s.n.], 1993. p. 147.

FOSTER, P. R. P.; BENINCASA, M.; LATANZE, R. J. Considerações para estimar os fluxos de radiação solar global para Jaboticabal - SP. In: CONGRESSO BRASILEIRO DE AGROMETEOROLOGIA, 4., 1985, Londrina.

Resumos... Londrina: [s.n.], 1985. p. 121.

FOSTER, P. R. P.; SANTOS, J. M. Considerações sobre o modelo de Angstron para estimativa dos fluxos de radiação solar global. In: CONGRESSO BRASILEIRO DE AGROMETEOROLOGIA, 4., 1985, Londrina. Resumos... Londrina: [s.n.], 1985. 
LAPERUTA FILHO, J.; LUNARDI, D. M. C.; JESUS, W. J. Modelos para estimativa da radiação solar global diária e horária por estação do ano em Botucatu-SP-Brasil. In: CONGRESSO BRASILEIRO DE AGROMETEOROLOGIA, 10., 1997, Piracicaba. Anais... Piracicaba: [s.n.], 1997. p. 194-195.

OMETTO, J. C. Estudo das relações entre radiação solar global, radiação líquida, insolação. 1968. 64 f.
Tese (Doutorado) - Escola Superior de Agricultura Luiz de Queiróz, Universidade de São Paulo, Piracicaba, 1968 .

SELlERS, W. D. Physical climatology. 5. ed. Chicago: The University of Chicago, 1974. 271 p.

TUBELIS, A.; NASCIMENTO, F. J. L. Meteorologia descritiva: fundamentos e aplicações brasileiras. São Paulo: Nobel, 1980. 374 p. 